\title{
A Novel Voltage Sensitivity Approach for the Decentralized Control of DG Plants
}

DOI:

10.1109/TPWRS.2017.2732443

\section{Document Version}

Accepted author manuscript

Link to publication record in Manchester Research Explorer

\section{Citation for published version (APA):}

Zhang, Z., Ochoa, LN., \& Valverde, G. (2017). A Novel Voltage Sensitivity Approach for the Decentralized Control of DG Plants. IEEE Transactions on Power Systems, PP(99). https://doi.org/10.1109/TPWRS.2017.2732443

\section{Published in:}

IEEE Transactions on Power Systems

\section{Citing this paper}

Please note that where the full-text provided on Manchester Research Explorer is the Author Accepted Manuscript or Proof version this may differ from the final Published version. If citing, it is advised that you check and use the publisher's definitive version.

\section{General rights}

Copyright and moral rights for the publications made accessible in the Research Explorer are retained by the authors and/or other copyright owners and it is a condition of accessing publications that users recognise and abide by the legal requirements associated with these rights.

\section{Takedown policy}

If you believe that this document breaches copyright please refer to the University of Manchester's Takedown Procedures [http://man.ac.uk/04Y6Bo] or contact uml.scholarlycommunications@manchester.ac.uk providing relevant details, so we can investigate your claim.

\section{OPEN ACCESS}




\title{
A Novel Voltage Sensitivity Approach for the Decentralized Control of DG Plants
}

\author{
Zedong Zhang, Luis F. Ochoa, Senior Member, IEEE, Gustavo Valverde, Senior Member, IEEE
}

\begin{abstract}
Renewable distributed generation (DG) is likely to be actively controlled in future distribution networks to mitigate voltage issues resulting from high penetrations. This requires understanding the corresponding dependencies between voltage magnitudes and DG active/reactive power outputs. One approach to compute these dependencies is to use classical sensitivity methods such as those based on the Jacobian matrix inverse. However, updating the latter involves extensive remote monitoring. This paper presents a novel approach to produce voltage sensitivities applying the Surface Fitting technique on data based solely on the knowledge of network characteristics; making it suitable for decentralized DG control. To assess the benefits, comparisons with classical methods are carried out using the 16-bus UK GDS test network (1-min resolution simulations) considering a decentralized voltage control algorithm that simultaneously caters for the active and reactive power outputs of a single DG plant. The robustness of the proposed approach is also investigated considering changes in network parameters. Finally, the use of coordinated time delays is proposed to cater for multiple DG plants. Comparisons with a centralized optimization demonstrate that the combined use of the proposed voltage sensitivity approach and decentralized control algorithm is an effective and implementable candidate to actively manage renewable DG plants.
\end{abstract}

Index Terms-Decentralized control, distributed generation, distribution networks, voltage sensitivity.

\section{INTRODUCTION}

E NVIRONMENTAL targets set by governments around the world are leading to high penetrations of small to medium-scale renewable generation, particularly wind and solar power. Large volumes of generation, however, can exceed the local demand and result in voltage and thermal issues among other technical problems [1]. The "Fit \& Forget" approach that refers to the traditional use of assets (such as lines and transformers) with limited or no control, in the context of distribution network planning, is used to meet maximum demand or generation requirements. However, to ensure that more renewable generation is cost-effectively connected to distribution networks, it is imperative to adopt a more active control of network elements and participants [2].

Approaches for the active control of distribution networks

Z. Zhang is with the School of Electrical and Electronic Engineering, The University of Manchester, UK. L. F. Ochoa is with the Department of Electrical and Electronic Engineering, The University of Melbourne, Australia, and with the School of Electrical and Electronic Engineering, The University of Manchester, UK. G. Valverde is with the School of Electrical Engineering, University of Costa Rica, Costa Rica. (emails: zedong.zhang@manchester.ac.uk, luis_ochoa@ieee.org, gustavo.valverde@ucr.ac.cr). can be categorized based on different aspects. From the perspective of requiring the corresponding network to be modelled during operation, control approaches can be categorized into model-based [3], [4] or model-free [5], [6]. From the perspective of dealing with uncertainties (e.g., renewable generation, communication issues, etc.), stochastic [7] and robust [8] approaches are among those proposed in the literature. Nonetheless, from a more practical perspective, the network coverage provided by the control approach is perhaps one of the most important aspects, particularly for Distribution Network Operators (DNOs). In this context, approaches are typically categorized into centralized, covering the whole network, and decentralized, covering only a local point. Centralized approaches have been widely investigated [9]. However, centralized methods require network observability, heavily relying on remote monitoring and the corresponding communication infrastructure [10]. In contrast, decentralized methods only need local information and no communication among local controllers. Therefore, the relative simplicity and low cost of the decentralized approaches make them attractive interim options before the implementation of more complex centralized schemes [4], [11].

Decentralized approaches for thermal management are relatively straightforward given that the excess of power flowing through an asset (or assets) can be mitigated by curtailing distributed generation (DG) by almost the same value. This 'direct' relationship, however, does not exist with voltages, making the corresponding management particularly challenging.

For voltage management, decentralized approaches available in the literature mainly focus on the regulation of the DG active and reactive power outputs. To specify the required adjustment, the voltage drop formula is applied in [12]. However, this is done considering the voltage at the other end of the corridor a constant value making this method not accurate enough for control purposes. A trial and error approach is adopted in [13] solving power flows for different DG active and reactive power outputs. Although this approach can be more precise, it requires extensive observation of the network.

Advanced capabilities of power electronic interfaces, such as the piece-wise linear volt/var and volt/watt curves provided by smart inverters [14], can also be used for decentralized voltage control purposes. However, defining the most adequate settings require either an offline analysis (that can be relatively complex [15]) or the adoption of conservative values. The performance of the latter will depend on the feeder layout, configuration and amount and location of PV [6].

Sensitivity approaches can be used to manage DG to regu- 
late voltages in a more accurate way. The traditional method of voltage sensitivity analysis is to use the Jacobian matrix inverse from a solved power flow case [16], [17]. Another approach known as perturb-and-observe analyzes first multiple power flows considering small changes in the DG active and/or reactive power outputs so as to extract the corresponding sensitivities [4]. However, during operation, updating the Jacobian matrix or running multiple power flows requires the network and its participants to be fully observable. Moreover, the resulting sensitivities obtained for a given period will not necessarily be valid for the next one (e.g., 1-min, 15-min) due to the inherent changes in load and generation.

The limited observability of distribution networks was considered more recently in [18] and [19]. An equivalent path between the control hub (e.g., substation) and the bus of interest (voltage to be regulated) is adopted in [18] using a constant conductance and susceptance. This approach, however, becomes less effective when loads or other DG plants are present in the corresponding corridor as they add significant variability to the equivalent path. In [19], a linear function is used to represent voltage changes and the corresponding coefficients are derived from historical data. With this linear approach, however, the resulting sensitivities become constant and therefore accuracy can be compromised, particularly when large variations occur in the power output of DG plants.

To overcome the above issues, this paper provides a novel approach by which voltage sensitivities are obtained based solely on the knowledge of network characteristics and therefore no remote monitoring is required. First, a large number of load and generation scenarios are created based on planning data, i.e., topology, conductor parameters as well as peak and minimum demand and generation. The Surface Fitting technique [20] is then applied to the resulting voltage and active/reactive power values in order to obtain the corresponding non-linear sensitivity functions. To update the sensitivity coefficient values, the only real-time information required by the proposed method corresponds to the local voltage and active/reactive power injections of the controlled DG plant.

To assess the performance of the proposed voltage sensitivity approach, a decentralized (local) voltage control algorithm that simultaneously caters for both the active and reactive power outputs of a single DG plant is adopted. Comparisons with classical methods are carried out using the 16-bus UK GDS test network and 1-min resolution demand and wind generation. The robustness of the method is also investigated considering potential differences between the adopted network model and the actual network, particularly in terms of R/X ratios and the upstream Thévenin equivalent reactance. Furthermore, to cater for multiple DG plants, this paper proposes a time-delay based decentralized control algorithm. Finally, to understand the extent to which the proposed control algorithm provides effective network management, a comparison with an ideal AC Optimal Power Flow (OPF) [3] is carried out.

This paper is structured as follows: Section II presents two sensitivity methods used in the literature and the proposed approach. Decentralized and centralized DG voltage control logics are described in section III. In section IV, the 16-bus
UK GDS test network is utilized to verify the three sensitivity methods. Further, the robustness of the proposed approach is investigated. Section $\mathrm{V}$ presents the control algorithm of multiple DG plants in a decentralized way as well as the comparison with an OPF-based centralized strategy. Finally, conclusions are drawn in section VII.

\section{Voltage SENSITIVITY ANALYSIS}

This section presents two approaches found in the literature that use voltage sensitivities produced during operation as well as the proposed method. Their advantages and observability requirements are discussed. A balanced network behavior is considered as this is common for the UK (region of interest). Examples of UK distribution networks can be found in [23]. The proposed method can also be applied to unbalanced networks which is discussed latter in section VI.

\section{A. Jacobian Matrix-Based Sensitivity Approach}

A classical approach to calculate voltage sensitivities during operation is to use the Jacobian matrix after solving a Newton Raphson power flow [16]. The voltage sensitivities (with respect to the magnitude of active $P$ and reactive power $Q$ ) are estimated by evaluating the Jacobian matrix inverse at a given operating point, as shown in (1). Here, $\boldsymbol{\theta}$ and $\boldsymbol{V}$ are the vectors of bus voltage angles and magnitudes, respectively. The magnitude of voltage variation $\Delta V_{D G}$ at the bus of interest (i.e., controllable DG plant) due to changes in active and/or reactive power can then be calculated using (2).

This approach, however, requires the voltage sensitivities to be updated every time the state of the network changes (e.g., demand and/or generation changes). This, in turn, requires new power flows and, hence, the full observation of the corresponding network. Therefore, the implementation of this approach requires further monitoring and communication infrastructures not yet common in distribution networks.

$$
\begin{gathered}
\boldsymbol{J}^{-1}=\left[\begin{array}{ll}
\frac{\partial \boldsymbol{\theta}}{\partial \boldsymbol{P}} & \frac{\partial \boldsymbol{\theta}}{\partial \boldsymbol{Q}} \\
\frac{\partial \boldsymbol{V}}{\partial \boldsymbol{P}} & \frac{\partial \boldsymbol{V}}{\partial \boldsymbol{Q}}
\end{array}\right] \\
\Delta V_{D G}=\frac{\partial V_{D G}}{\partial P_{D G}} * \Delta P_{D G}+\frac{\partial V_{D G}}{\partial Q_{D G}} * \Delta Q_{D G}
\end{gathered}
$$

\section{B. Perturb-And-Observe Power Flow-Based Approach}

An approach that uses power flow results instead of the Jacobian matrix is proposed in [4] to calculate voltage sensitivities during operation. It considers the current state of the network and two power flows with a small change in the active (or reactive) power at the bus of interest. Let $V_{D G}$ be the voltage at the bus of a controllable DG plant with $Q_{D G}$ MVar of reactive power output. To extract the corresponding voltage sensitivity at a given instant, a snapshot power flow is carried out considering the same demand and generation levels but with a reactive power of " $Q_{D G}+1$ " MVar. The resulting voltage at the bus of interest, $V_{D G^{\prime}}$, is then used to calculate the sensitivity, as shown in (3). 


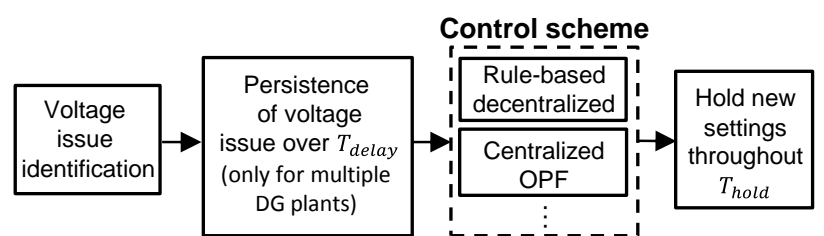

Fig. 1. General process for the voltage control of an individual DG plant

$$
\frac{\partial V_{D G}}{\partial Q_{D G}}=\frac{V_{D G^{\prime}}-V_{D G} p . u}{1 M v a r / S_{\text {base }}}
$$

Similarly to the Jacobian-based approach, the use of perturb-and-observe implies the need to fully observe the network as well as the continuous update of the sensitivities.

\section{Fitting Function-Based Sensitivity Approach}

The first two approaches focus on the extraction of the voltage sensitivities during operation to then calculate the active and/or reactive power needed to change the voltage at the bus of interest. These sensitivities, however, require continuous observation of the network to carry out the updates.

To eliminate the need of remote monitoring, the proposed approach uses a large number of power flows based on planning data to produce a non-linear function that describes the dependencies between the voltage of the bus of interest, $V_{D G}$, and the DG power injections, $P_{D G}$ and $Q_{D G}$, i.e., $V_{D G}=$ $f\left(P_{D G}, Q_{D G}\right)$. The resulting sensitivities, obtained by calculating the partial differential equations $\partial V_{D G} / \partial P_{D G}$ and $\partial V_{D G} /$ $\partial Q_{D G}$, can then be used locally whilst catering for all the potential demand and generation changes across the network.

First, considering only planning data such as topology and conductor parameters, as well as location, rated capacity and type of demand and generation, thousands of demand/generation scenarios are created to cover as many of the possible states of the network, i.e., to cover changes in load and all generators throughout the day, week, month or year. To do this, demand and generation levels are created uniformly from 0 to $100 \%$ of their power capacities, respectively. Power factor settings for the DG plant are also identified uniformly within the available range. Then, the possible scenarios are created by combining the different demand/generation levels and power factor settings. After power flow calculations for all scenarios, a three-dimensional space is then established in which the samples are the values of $V_{D G}$ (dependent variable) and the corresponding values of $P_{D G}$ and $Q_{D G}$ (independent variables). The Surface Fitting technique [20] is applied to produce the fitting function $V_{D G}=f\left(P_{D G}, Q_{D G}\right)$. Finally, this is used to obtain the voltage sensitivities with (4) and (5).

$\frac{\partial V_{D G}}{\partial P_{D G}}=\frac{\partial f\left(P_{D G}, Q_{D G}\right)}{\partial P_{D G}}$ (4) $\frac{\partial V_{D G}}{\partial Q_{D G}}=\frac{\partial f\left(P_{D G}, Q_{D G}\right)}{\partial Q_{D G}}$

Consequently, the proposed approach makes it possible to continuously update the voltage sensitivities by only using information at the bus of interest (i.e., $P_{D G}$ and $Q_{D G}$ ), i.e., only local monitoring is required. Furthermore, by describing these sensitivities as functions, more accurate values can be obtained than using simpler approaches such as lookup tables (in which sensitivities are represented by discrete values for different combinations of $P_{D G}$ and $Q_{D G}$ ).

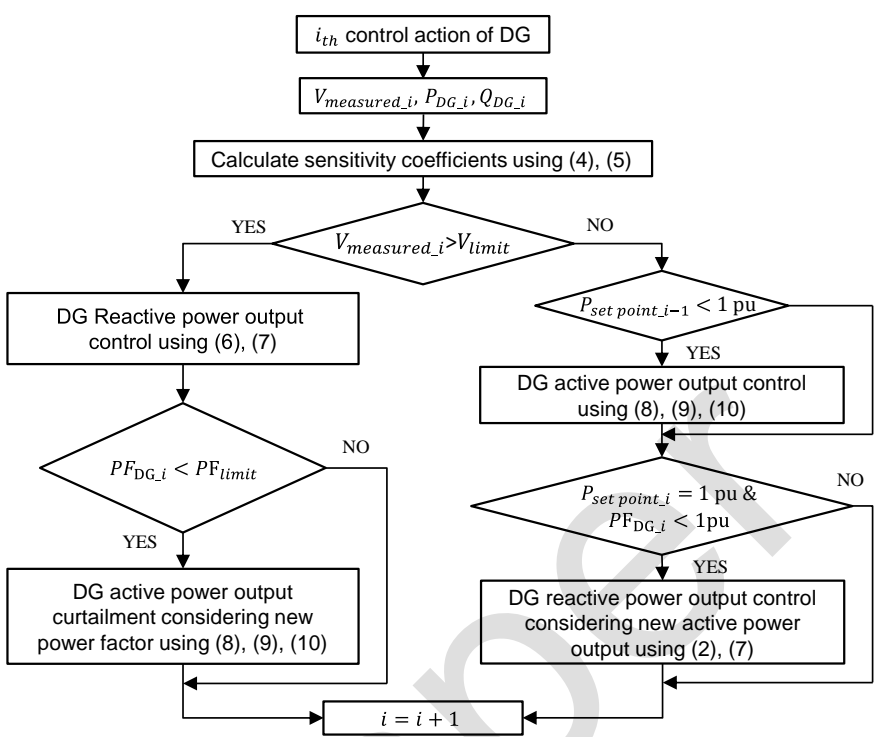

Fig. 2. Proposed rule-based decentralized voltage control logic

It should be noted that the Surface Fitting technique considers the samples to be normally distributed across the investigated space. However, if that is not the case, the resulting fitting function will tend to be more accurate where the density of samples is higher [21].

An alternative to the proposed approach is to obtain sets of Jacobian matrix-based voltage sensitivities for multiple demand and generation scenarios and then use the Surface Fitting technique to produce the corresponding sensitivity functions that would replace (4) and (5). Although the resulting accuracy might be similar, the proposed approach is simpler and faster as it does not require calculating any element of the inverted Jacobian matrix after each power flow is solved.

It is worth highlighting that the production of demand/generation scenarios can also be done considering historic data or realistic Probability Density Functions (PDFs). Such approaches, however, could result in sensitivities biased towards specific scenarios that are more frequent and might not necessarily work for less frequent scenarios. Therefore, the benefit of mapping as many scenarios as possible, as proposed, is that the resulting sensitivities are likely to be more generic and, as such, they will work for most scenarios.

\section{DeCEnTRALIZED AND CENTRALIZED DG CONTROL LOGIC}

The effectiveness of different sensitivity approaches can be assessed once embedded into a control scheme and applied to a distribution network. For this purpose, here it is proposed a rule-based decentralized control logic to manage voltage rise issues at the connection point of a single DG plant. This is then extended to multiple DG plants by adopting time delays. Further, to assess the extent to which the decentralized control logic provides effective DG control, an ideal AC OPF-based centralized approach is presented to be used for comparisons. A balanced network behavior is considered for simplicity.

\section{A. Voltage Control Process}

Fig. 1 shows the proposed process for the voltage control of an individual DG plant. This is triggered when a voltage issue at the DG connection point (rule-based decentralized ap- 
proach) or at any point in the network (AC OPF-based centralized approach) is detected. If there is only one DG plant being controlled, new settings are found according to the adopted control scheme. To reduce the volume of control actions, the new settings are held for a time period, $T_{\text {hold }}$.

For multiple DG plants, coordination of their actions is required when adopting the decentralized approach. This is achieved by considering different time delays, $T_{\text {delay }}$, immediately after a voltage issue is identified, as well as different values for $T_{\text {hold }}$. Further details are presented in section III-C.

\section{B. Decentralized Control Logic - Single DG Plant}

The flowchart of the decentralized DG control logic is shown in Fig. 2. When the DG control is triggered (control action $i$ ), measurements of DG active/reactive power outputs $\left(P_{D G_{-} i}, Q_{D G_{-} i}\right)$ are used to calculate the sensitivity coefficients using (4) and (5). The voltage at the DG connection point, $V_{\text {measured_i }}$, is compared to the pre-defined voltage limit, $V_{\text {limit }}$. The latter must be set to a value that is low enough to decrease the occurrence of voltage swells (e.g., due to sudden gusts) that exceed the statutory limit of the network but also high enough to ensure an adequate harvesting of energy.

1) DG Control when the Voltage Limit is Exceeded

If the measured voltage exceeds the limit, the DG reactive power output is controlled first by adjusting the power factor setting. To achieve this, the required reactive power absorption is calculated using (2) and assuming $\Delta P_{D G_{-} i}$ is zero (i.e., no curtailment), as follows:

$$
\Delta Q_{D G_{-} i}=\frac{V_{\text {limit }}-V_{\text {measured } \_} i}{\partial V_{D G_{-} i} / \partial Q_{D G_{-} i}}
$$

The corresponding power factor is obtained using (7):

$$
P F_{D G_{-} i}=\frac{P_{D G_{-} i}}{\sqrt{P_{D G_{-} i}{ }^{2}+\left(Q_{D G_{-} i}+\Delta Q_{D G_{-} i}\right)^{2}}}
$$

If the power factor, $P F_{D G_{-}}$, is smaller than the limit of the DG plant, $P F_{\text {limit }}$, then the latter is used as the new setting. In such case, the resulting reactive power absorption will not be enough to reduce the voltage rise, hence active power curtailment is required. This is calculated using (8):

$$
\Delta P_{D G_{-} i}=\frac{V_{\text {target }}-V_{\text {measured } \_i}-\frac{\partial V_{D G_{\_} i}}{\partial Q_{D G_{\_} i}} * \Delta Q_{D G_{-} i}}{\partial V_{D G_{-} i} / \partial P_{D G_{-} i}}
$$

where

$$
\Delta Q_{D G_{-} i}=\left(P_{D G_{-} i}+\Delta P_{D G_{-} i}\right) * \sqrt{\frac{1}{P F_{\text {limit }}{ }^{2}}-1}-Q_{D G_{-} i}
$$

Finally, the DG output set point is obtained as follows:

$$
P_{\text {set point } \_i}=\frac{P_{D G_{-} i}+\Delta P_{D G_{-} i}}{P_{D G_{-} i} / P_{\text {set point } \_i-1}}
$$

When the DG active power set point (10) and the power factor setting (7) are defined, they are implemented immedi-

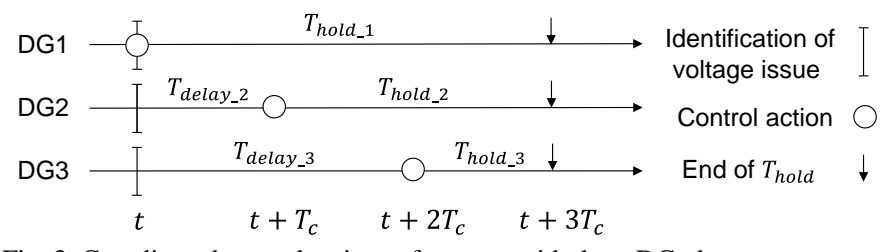

Fig. 3. Coordinated control actions of a group with three DG plants

ately by the DG plant.

It should be noted that the assumption of constant voltage sensitivity values during a given control action will not necessarily achieve the desired outcome, as the sensitivities are actually state-dependent and the control actions themselves have already changed the state of the network. This is why the volt age is regularly checked so as to provide more opportunities to trigger further control actions. Nonetheless, in practice, the variability brought by renewable DG plants is likely to create larger changes in the state of the network than the changes brought by this assumption.

It is also worth noting that due to the power factor limit, $P F_{\text {limit }}$, any complex power resulting from the absorption of reactive power (to lower voltages) will be limited to what is possible based on the active power output of the DG plant. Therefore, the corresponding lines are not expected to see a dramatic increase their loading. For instance, 0.95 leading/lagging power factor capabilities will have a maximum increase in complex power output of 5.26\% if moving from unity power factor to the 0.95 value. Given the focus of this work, it is assumed that the existing lines can cope with the complex rated output of the DG plants.

\section{2) DG Control when the Voltage Limit is Not Exceeded}

As shown in Fig. 2, if the measured voltage is smaller than the pre-defined voltage limit (i.e., voltage headroom is available), more DG active power output can be injected. In addition, reactive power absorption, if any, can also be reduced. To ensure the most adequate energy harvesting from the DG plant, first it is checked whether curtailment has been applied (i.e., $P_{\text {set point } \_i-1}<1 p . u$.). The potential extra power injection is calculated using (8) considering also the change in reactive power (9). The set point is given by (10).

If after the above control, the DG plant reaches its nominal active power set point (i.e., $P_{\text {set point } i}=1 p$. u., which indicates that additional voltage headroom is available) and the DG power factor is smaller than 1 p.u., then the reactive power absorbed by the DG plant can be reduced. This is obtained using (2) and considering the $\Delta P_{D G_{-} i}$ calculated above. The power factor setting is calculated using (7).

\section{3) Simultaneous $P-Q$ Control Logic}

It is important to highlight that the proposed control logic caters for both the DG active and reactive power outputs, i.e., new settings are assigned simultaneously. Most sensitivitybased methods available in the literature use separate sensitivities for active and reactive power and thus sequential control actions are needed (i.e., one for reactive power and then for active power). The proposed fitting function-based approach finds the most adequate settings for both using only one equation. This reduces control actions (both are implemented simultaneously) and can help improving the overall performance. 


\section{Time Delays - Multiple DG Plants}

The uncoordinated decentralized operation of multiple DG plants can make the proposed control logic ineffective given that, in the event of a voltage issue detected simultaneously by multiple DG plants within the same feeder, a DG plant would take actions without considering that others might do the same. This, in turn, would result in further corrective actions, poor voltage management, and higher levels of curtailment.

To achieve the adequate decentralized operation of multiple DG plants, and given that no communication is available, here it is proposed to coordinate the timing of their actions. For this purpose, delays before $\left(T_{\text {delay }}\right)$ and after $\left(T_{\text {hold }}\right)$ control actions are adopted. Different values are allocated to each DG plant to ensure control actions do not occur at the same time.

Depending on the topology of the network, the injections from one DG plant might not necessarily influence all others (in terms of voltages). Therefore, the values of $T_{\text {delay }}$ and $T_{\text {hold }}$ are defined per group of DG plants which are identified considering this influence. For instance, DG plants connected to the same feeder would correspond to the same group.

Within the same DG group, the values of $T_{\text {delay }}$ and $T_{\text {hold }}$ are defined considering that the further a DG plant is from a voltage regulation point (e.g., substation with on-load tap changer-fitted transformers), the higher the priority of curtailment, i.e., faster control action and, thus, smaller $T_{\text {delay }}$. This also means larger $T_{\text {hold }}$ to allow time for the control actions of other DG plants within the group.

Therefore, for a group with $m$ DG plants ranked from 1 to $m$ according to their curtailment priorities, the values for $T_{\text {delay }}$ and $T_{\text {hold }}$ for a DG plant with rank of $n$ are calculated using (11) and (12), respectively, where $T_{c}$ is the time considered acceptable between two control actions.

$$
\begin{aligned}
& T_{\text {delay_n}}=(n-1) \times T_{c} \\
& T_{\text {hold_n } n}=(m-n+1) \times T_{c}
\end{aligned}
$$

To illustrate this, Fig. 3 shows the timings of actions of a group with $m=3$ DG plants that, due to the generation and/or demand conditions within the same feeder, have detected voltage issues simultaneously at time $t$. Assuming DG1 has the first priority, followed by DG2 and DG3, the corresponding $T_{\text {delay }}$ and $T_{\text {hold }}$ values are $0, T_{c}, 2 T_{c}$, and $3 T_{c}, 2 T_{c}, T_{c}$, respectively. In this way, when voltage issues are simultaneously detected by multiple DG plants, the $T_{\text {delay }}$ and $T_{\text {hold }}$ allow them to have control actions one after the other following the pre-defined priority until the voltage issues are solved. Consequently, the DG actions do not require any level of synchronization (i.e., time aligning) as this is done through coordination.

It should be highlighted that the suggested allocation of delays is not necessarily the most equitable as it will penalize the DG plants at the furthest locations. However, this prioritization of curtailment was only used for illustration purposes as many other strategies do exist [22]. Furthermore, the proposed time delays are flexible and, therefore, the DNO can determine them considering not only technical aspects but also economic and/or contractual ones. From the technical perspective, time delays should be long enough that none of DG actions inter- rupt each other but should also be tuned to solve issues as fast as required (considering the response time of each DG plant). From the economic and/or contractual perspective, time delays can also be defined following the most adequate priority agreed by DG owners and the DNO, such as Last In First Out (LIFO) or Shedding Rota [22]. For the LIFO, for instance, the highest priority of curtailment would be given to the DG plant that connected to the network at last, and so on. However, other approaches, such as Pro Rata [22], require adapting the control logic to reflect the share of the corresponding DG plant when control actions are needed.

\section{AC Optimal Power Flow}

In this centralized control scheme, the optimal active/reactive power output of DG plants and the tap ratios of transformers are calculated by the AC OPF which requires the full observability of the network. To maximize the total DG active power output $p$ of a set of generators $G$ (indexed by $g$ ), the objective function of the AC OPF is given below:

$$
\max \sum_{g \in G} p_{g}
$$

This objective is subject to the typical AC OPF constraints (i.e., Kirchhoff's voltage and current laws), DG capacity and voltage limits. For each line $l$ of a set of lines $L$, the active and reactive power injections $f_{l}^{(1,2),(P, Q)}$ at the start and end busses (denoted by 1 and 2 ) of the line are calculated according to the Kirchhoff's voltage law assuming three phases are balanced:

$$
f_{l}^{(1,2),(P, Q)}=f_{l, K V L}^{(1,2),(P, Q)}(V, \theta)
$$

where $V$ and $\theta$ are the voltage magnitude and phase angle respectively. In terms of Kirchhoff's current law, the balance of active and reactive power at each bus ( $\operatorname{set} B$ indexed $b$ ), are formulated as follows:

$$
\begin{aligned}
& \sum_{d \in D} p_{d}+\sum_{l \in L} f_{l}^{(1,2), P}=\sum_{x \in X} p_{x}+\sum_{g \in G} p_{g} \\
& \sum_{d \in D} q_{d}+\sum_{l \in L} f_{l}^{(1,2), Q}=\sum_{x \in X} q_{x}+\sum_{g \in G} q_{g}
\end{aligned}
$$

where $\left(p_{x}, q_{x}\right)$ are the active and reactive power from the grid (set $X$ indexed by $x) ;\left(p_{d}, q_{d}\right)$ are the active and reactive demands (set $D$ indexed $d$ ), respectively.

In the context of this work, the reactive power of controllable DG plants is also dispatchable (i.e., can operate at different power factors). For this purpose, the power factor angle for each controlled DG plant, $\emptyset_{g}$, is considered as a variable and it is required to operate within the DG plant's capability.

Transformers equipped with voltage regulation devices, will have the voltages at the primary sides divided by the tap ratio $T_{l}$. Given that the AC OPF is formulated with continuous variables, the resulting tap ratios need to be rounded up to the nearest discrete value that represents a feasible position.

\section{Simple Case Study - Single DG Plant}

In this section, the previously presented sensitivity approaches are applied to a 16-bus UK generic distribution net- 


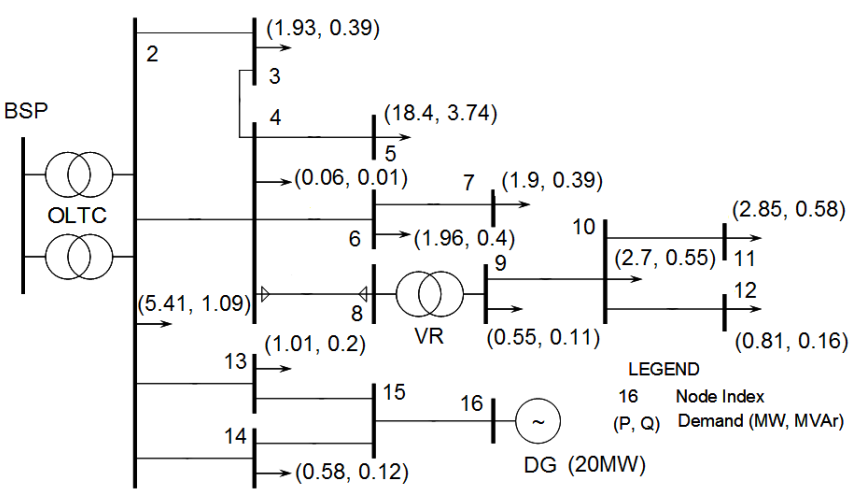

Fig. 4. UK GDS Simplified EHV1 Network [23].

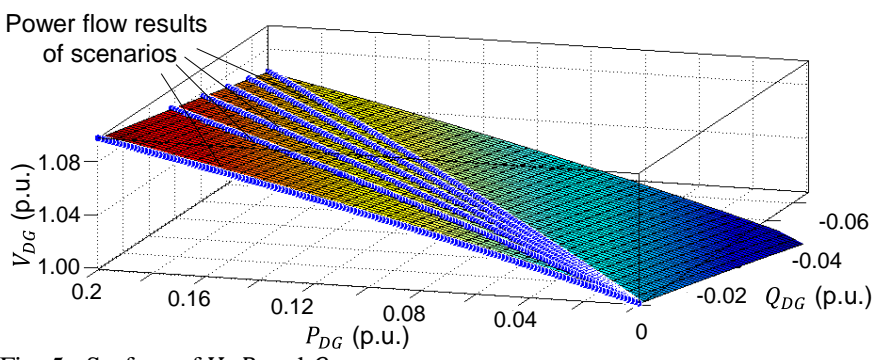

Fig. 5. Surface of $V, P$ and $Q$.

work. First, the adequacy of different number of scenarios to be used by the fitting function-based approach is investigated. Then, a comparison of the corresponding coefficients is carried out. The performance of each approach, in terms of their ability to manage voltages, is assessed considering the proposed decentralized control logic. Finally, the robustness of the proposed sensitivity method is also investigated considering potential differences between the parameters of the network model used to produce the sensitivities and those of the network where the resulting DG settings are applied to.

\section{A. UK GDS Simplified EHVI Network}

The network studied in this section is the UK Generic Distribution System (GDS) Simplified 16-bus EHV1 Network [23]. The topology is shown in Fig. 4. This network has a peak demand of $18.4 \mathrm{MW}$ and is supplied by two $30 \mathrm{MVA}$ $132 / 33 \mathrm{kV}$ transformers. The voltage statutory limits in the $33 \mathrm{kV}$ circuit are $\pm 6 \%$ of nominal. The OLTC at the substation and the voltage regulator (VR in Fig. 4) have voltage targets of 1.036 and 1.03 p.u. at their secondary sides, respectively, and use a voltage deadband of $\pm 0.5 \%$. For this case study a modern $20 \mathrm{MW}$ wind farm with a 0.95 leading/lagging power factor capability (available also during full active power output [24]) is connected to bus 16 .

\section{B. Voltage Sensitivity - Influence of Scenarios}

For the proposed approach, a large number of demand and generation scenarios are first created to realistically cover most of the possible network states. In this case, 150 demand and 150 generation levels are identified (from 0 to $100 \%$ demand/generation) as well as 6 power factors settings for the wind farm (0.95-0.99 leading, in 0.01 steps, and unity), i.e., a total of $150 \times 150 \times 6=135,000$ scenarios.

Power flows are calculated for each scenario and the resulting voltages at bus $16, V_{D G}$, together with the corresponding

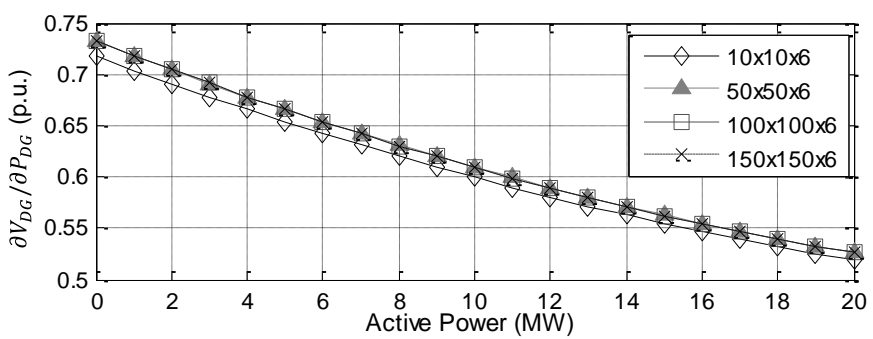

Fig. 6. Sensitivity coefficients for unity power factor derived from four sets: $10,50,100$, and 150 demand/generation levels and 6 power factor settings.

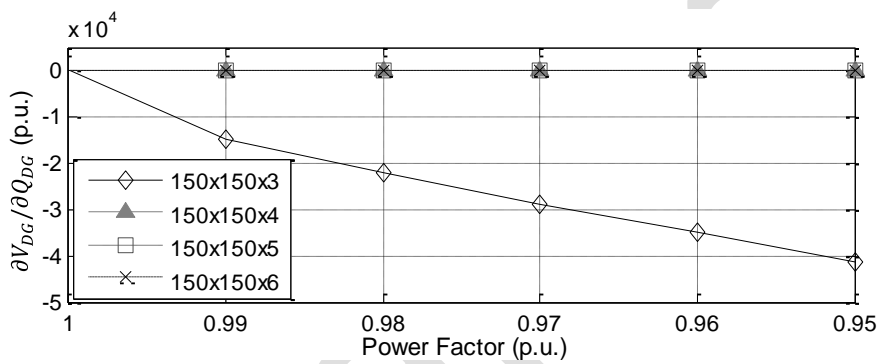

Fig. 7. Sensitivity coefficients for peak generation derived from four sets: 150 demand/generation levels and 3,4,5, and 6 power factor settings.

DG active and reactive power outputs (i.e., $P_{D G}$ and $Q_{D G}$ ), are then used to produce a three-dimensional surface as shown in Fig. 5. Finally, the Surface Fitting technique is applied to produce the corresponding third-order non-linear fitting function (17), where $a_{j k}$ represents the constant weights; $j$ and $k$ are the indices. This process can be achieved using non-linear regression analysis available in software packages such as Matlab [25] where usually numerical optimization algorithms are applied to determine the corresponding weights. With this function, the voltage sensitivity coefficients with respect to the active and reactive power of the wind farm $\left(\partial V_{D G} / \partial P_{D G}\right.$, $\left.\partial V_{D G} / \partial Q_{D G}\right)$ can be obtained by calculating the corresponding differentiations.

$$
V_{D G}\left(P_{D G}, Q_{D G}\right)=\sum_{j=0}^{3} \sum_{k=0}^{3} a_{j k} P_{D G}{ }^{j} Q_{D G}{ }^{k}
$$

The adequacy of the 135,000 scenarios considered in this work can only be assessed by comparing the resulting voltage sensitivity coefficients against those obtained with different sets of scenarios. For this purpose, three sets of 10, 50 and 100 demand and generation levels each with 6 power factor settings, i.e., a total of $600(10 \times 10 \times 6), 15,000(50 \times 50 \times 6)$, and 60,000 (100 x $100 \times 6)$ scenarios, respectively, are considered. Fig. 6 shows the coefficients with respect to the active power of the wind farm for unity power factor, i.e., $Q_{D G}$ is effectively removed from (17) and therefore a comparison in the two-dimensional space is possible. This figure highlights that the sensitivity coefficients for the set with 600 scenarios is different from the others who have similar values. This is also verified in Fig. 8(a) where the corresponding Mean Squared Error (MSE) between the coefficients of consecutive sets for different power factors (from 0.95 leading to 1.0 in 0.01 steps) are shown. Crucially, for any power factor, the MSE gets smaller and more stable as the number of scenarios gets larger. Consequently, it can be concluded that, in this case, 150 demand and generation levels lead to accurate coefficients and 


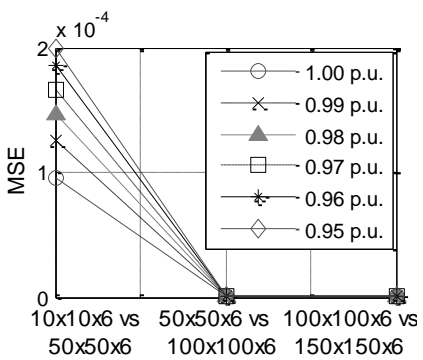

(a)

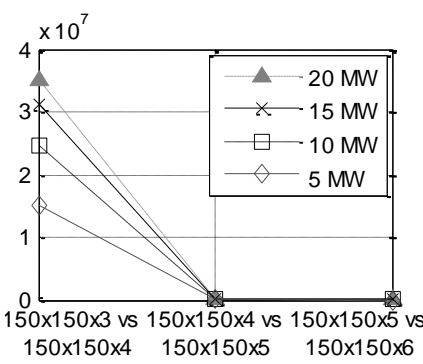

(b)

Fig. 8. MSE between sensitivity coefficients derived from different (a) sets of demand and generation levels (b) sets of power factors settings.

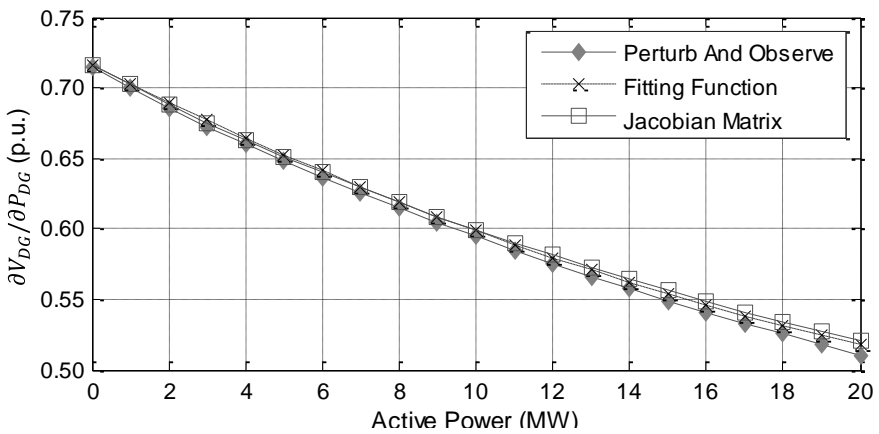

Fig. 9. Comparison of the voltage sensitivity of bus 16 with respect to active power considering different approaches.

that further granularity would not bring significant benefits.

Considering 150 demand and generation levels, the voltage sensitivity coefficients derived from four sets combining $3,4,5$ and 6 power factor settings are shown in Fig. 7 for the rated active power output of the wind farm, i.e., making $P_{D G}$ in (15) a constant equal to $20 \mathrm{MW}$. It can be noticed that with only 3 power factor settings the sensitivity coefficients are significantly different from the other sets. This is also proved by the MSE values considering various power injections from the wind farm (from 5 to $20 \mathrm{MW}$ in $5 \mathrm{MW}$ steps) shown in Fig. 8(b). The MSE gets smaller and more stable for a larger number of scenarios regardless the power injection. Thus, 6 power factors settings are adopted to ensure higher accuracy.

\section{Voltage Sensitivity - Comparison}

To assess the accuracy of the proposed fitting functionbased approach (with 135,000 scenarios), the voltage sensitivity coefficients with respect to active power output $\left(\partial V_{D G} /\right.$ $\left.\partial P_{D G}\right)$ are compared with those obtained using the Jacobian matrix and perturb-and-observe approaches. Fig. 9 shows the corresponding values considering minimum load (specified for the other two approaches) and unity power factor. The results demonstrate that the fitting function reaches similar values to those of the Jacobian matrix and is even better than the perturb-and-observe. Crucially, this accuracy is achieved without the need of full network observability.

\section{Performance of the Voltage Sensitivity-Based Control}

Although the three sensitivity approaches have similar coefficient values and behaviors, the actual performance in terms of voltage management needs to be assessed. To do this, the proposed rule-based decentralized DG control logic as well as the AC OPF are used considering 1-min resolution demand and wind power profiles (i.e., normalized available resource)

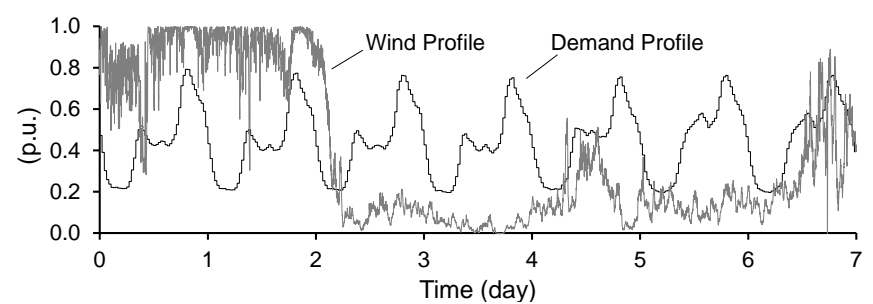

Fig. 10. Normalized demand and wind power profiles, first week Feb 2010
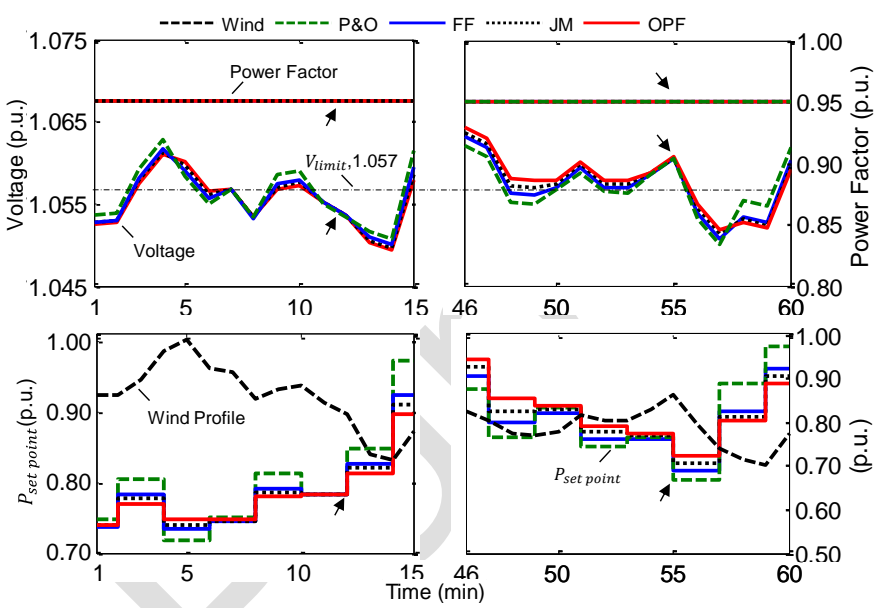

Fig. 11. Voltage profiles at bus 16; power factor set points, active power set points for wind farm using control schemes (OPF: Optimal Power Flow, JM: Jacobian Matrix, FF: Fitting Function, P\&O: Perturb And Observe.)

from the North of England for the first week of February 2010 (Fig. 10). The voltage limit for decentralized and centralized approaches is set to 1.057 p.u. - close to the statutory limit of 1.06 p.u. Control actions are triggered when the voltage of the last minute violates this limit. New settings are then held for 2 minutes $\left(T_{\text {hold }}\right)$. The analysis is carried out using OpenDSS [26], MS Excel VBA [27], and AIMMS [28]. The existing lines are assumed able to cope with the complex rated output of the DG plants as explained in section III-B.

The resulting voltage profiles at bus 16 (wind farm connection point) and the set points of the wind farm are presented in Fig. 11 for the first and last 15-minute blocks between 1:00 am to 2:00 am on $1^{\text {st }}$ Feb. Within this period demand changes from one minute to the next are small (maximum of $0.14 \mathrm{MW}$ ) while changes in the available wind resource are significant (maximum of 0.13 p.u., equivalent of $2.58 \mathrm{MW}$ ). Because of these characteristics, the controllers are mainly addressing voltage issues brought by the wind farm.

At minute 12, the four approaches reach almost the same voltage (1.053 p.u.) as well as active power and power factor set points ( 0.78 and 0.95 p.u., respectively). Given that at this point the voltage is below $V_{\text {limit }}$ and curtailment has been applied, new settings are required to increase the harvesting of wind energy. However, the different accuracies of the control approaches result in different active power set points and therefore in different voltages at minute 13. The AC OPF has the highest active power set point and the resulting voltage is the closest to $V_{\text {limit }}$. The second best approach is the proposed fitting function followed by the Jacobian matrix and the perturb-and-observe approaches. These new settings are kept through $T_{\text {hold }}$ until minute 14. 
TABLE I - PERFORMANCE: DECENTRALIZED JACOBIAN MATRIX, Perturb-And-ObSERve, Fitting Function ANd CEnTRALized AC OPF

\begin{tabular}{|c|c|c|c|}
\hline \multirow[b]{2}{*}{ Control Methods } & \multicolumn{2}{|c|}{ Performance Metrics } & \multirow{2}{*}{$\begin{array}{c}\text { Required } \\
\text { Real-time } \\
\text { Information }\end{array}$} \\
\hline & $\begin{array}{c}\text { BS EN50160 } \\
\text { Compliance } \\
(\%)\end{array}$ & $\begin{array}{c}\text { Capacity } \\
\text { Factor } \\
\text { (p.u. })\end{array}$ & \\
\hline $\begin{array}{l}\text { Centralized } \\
\text { AC OPF }\end{array}$ & 96.924 & 0.3216 & $\begin{array}{l}\text { Full network } \\
\text { observability }\end{array}$ \\
\hline $\begin{array}{l}\text { Decentralized } \\
\text { Fitting Function }\end{array}$ & 96.726 & 0.3212 & $V_{D G}, P_{D G}, Q_{D G}$ \\
\hline $\begin{array}{c}\text { Decentralized } \\
\text { Jacobian Matrix }\end{array}$ & 95.933 & 0.3212 & $\begin{array}{l}\text { Full network } \\
\text { observability }\end{array}$ \\
\hline $\begin{array}{c}\text { Decentralized } \\
\text { Perturb-And-Observe }\end{array}$ & 95.833 & 0.3204 & $\begin{array}{l}\text { Full network } \\
\text { observability }\end{array}$ \\
\hline
\end{tabular}

TABLE II - RoBUSTNESS: DECENTRALIZED JACOBIAN MATRIX, FITTING FUNCTION AND CENTRALIZED AC OPF

\begin{tabular}{c|c|c|c|c|c}
\hline \multirow{2}{*}{ Case } & \multirow{2}{*}{$\begin{array}{c}\text { TER } \\
\text { (p.u.) }\end{array}$} & \multirow{2}{*}{$\begin{array}{c}\text { Average } \\
\boldsymbol{R} / \boldsymbol{X} \\
\end{array}$} & (p.u.) & \multicolumn{2}{|c}{ BS EN50160 Compliance $(\%)$} \\
\cline { 4 - 6 } & & $\begin{array}{c}\text { Jacobian } \\
\text { Matrix }\end{array}$ & $\begin{array}{c}\text { Fitting } \\
\text { Function }\end{array}$ & $\begin{array}{c}\boldsymbol{A C} \\
\boldsymbol{O P F}\end{array}$ \\
\hline 1 & 0 & 1.05 & 95.833 & 96.726 & 96.925 \\
\hline 2 & 0.25 & 1.05 & 95.933 & 96.825 & 97.024 \\
\hline 3 & 0.5 & 1.05 & 96.032 & 96.925 & 97.222 \\
\hline 4 & 0.75 & 1.05 & 96.131 & 97.024 & 97.321 \\
\hline 5 & 0 & 1.26 & 94.544 & 95.437 & 95.635 \\
\hline 6 & 0 & 0.84 & 96.726 & 97.421 & 97.619 \\
\hline
\end{tabular}

Very similar voltages (1.061 p.u.) as well as active power and power factor set points (0.86 and 0.95 p.u., respectively) are reached by the four approaches at minute 55. Although all the approaches solve the voltage rise problem in minute 56, Fig. 11 demonstrates that yet again the proposed fitting function is the second best approach as its active power set point is the closest to that obtained by the AC OPF.

The weekly performance metrics for the four approaches are presented in Table I. Even though the differences among the approaches are small, the numerical results confirm that the fitting function-based approach performs slightly better than the Jacobian matrix and the perturb-and-observe considering voltage compliance with the BS EN50160 [29] standard (95\% of 10 -min voltage magnitudes must be within $\pm 6 \%$ of nominal) and the capacity factor (i.e., energy harvesting).

The higher performance of the proposed approach is due to the conservative nature of the corresponding coefficients during curtailment. Indeed, as it can be seen in Fig. 9, the fitting function-based coefficients are slightly lower than those from the Jacobian matrix particularly during high generation outputs (when curtailment is needed). This, in turn, results in larger curtailment, and lower voltages. In contrast, during lower generation, the coefficients from both sensitivity methods are almost the same, resulting in very similar DG active power output adjustments and, thus, voltage increases. The accuracy of the coefficients obtained by the fitting function is explained by the density of the corresponding samples. As it can be seen in Fig 5, closer to the origin for both $P_{D G}$ and $Q_{D G}$ the triangular-shaped area of samples has a higher density and therefore results in more accurate coefficients. Conversely, the further from this origin (higher generation), the density of samples decreases, thus leading to less accurate coefficients.

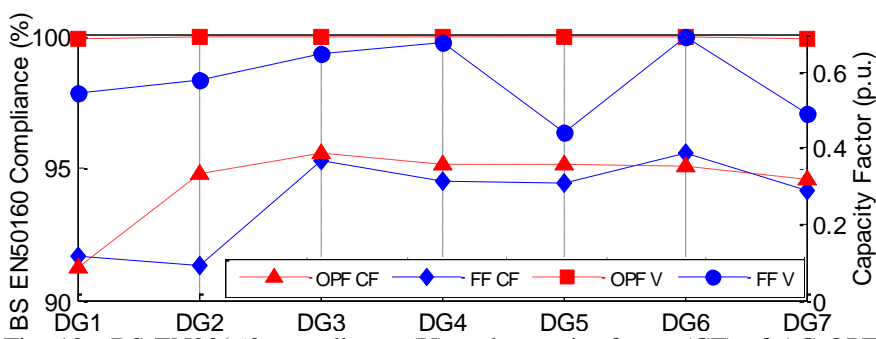

Fig. 12. BS EN20160 compliance (V) and capacity factor (CF) of AC OPF and Fitting Function-based decentralized control

\section{E. Analysis of Robustness}

In the previous analysis the parameters of the network model used to produce the proposed sensitivity function are considered to be equal to those of the network in which the resulting DG settings are applied to. However, in practice, network parameters are likely to differ due to changes in temperature, loading, etc. To understand the extent of the robustness of the proposed fitting function-based approach in the presence of inaccuracies, as well as that of the Jacobian matrix-based approach and the AC OPF, changes in the network to which settings are applied are investigated. Specifically, these changes consider line impedances to have 1.26 and 0.84 p.u. of average $R / X$ ratio ( $\pm 20 \%$ of the 1.05 ratio found in the original 16-bus UK GDS network) and upstream Thevenin Equivalent Reactance (TER) values of $0.25,0.5$ and $0.75 \mathrm{p} . \mathrm{u}$ (different from the ideal 0 p.u. of the original network).

The performance of the three approaches considering the changes described above are presented in Table II; the original network model is used in all cases to produce the new DG settings. In the cases 2, 3 and 4, where different TER values are adopted for the network to which the DG settings are applied (the 'real' network), the three approaches still satisfy the BS EN20160 standard and perform even better than the case without mismatch (case 1). This is because the larger the TER, the lower the voltages across the network, which in turn makes the DG settings produced with a 0 p.u. of TER conservative, resulting in less voltage rise issues. With mismatches in the $R / X$ ratio (cases 5 and 6 ), the three approaches continue to achieve good results. It can be seen, however, that a higher $R / X$ ratio mismatch leads to more voltage rise issues (case 5). This is due to the fact that the estimated curtailment is not enough when applied on the 'real' network (the settings are calculated assuming a lower $R / X$ in which DG reactive power absorption results in less curtailment).

Although the three approaches are affected by the investigated mismatches, they can be considered robust enough when applied to real networks. More importantly, the proposed approach continues to outperform the Jacobian matrix and have results similar to the $\mathrm{AC} \mathrm{OPF}$.

\section{Full Case Study - Multiple DG Plants}

The analysis is now extended to the control of multiple DG plants using the same UK GDS network but with seven wind farms. The performance of the proposed decentralized control logic, now adopting coordinated time delays for multiple DG plants, is compared with the benchmark AC OPF.

The seven wind farms (DG1 to DG7) are connected to bus- 
es $16,15,14,11,12,10$, and 7 with capacities of $8,10,12$, $2.5,2.5,2$, and $12 \mathrm{MW}$, respectively. Three different wind profiles, WP1, WP2 and WP3, are adopted: WP1 is the same wind profile shown in Fig. 8, whereas WP2 and WP3 are based on WP1 but with shifts of 1 and 2 hours behind, respectively. The profiles are allocated considering the locations of the buses: 7, 11, and 16 use the WP1 profile; 12 and 15 use the WP2 profile; and, 10 and 14 uses the WP3 profile. The corresponding scenarios were produced considering groups of DG plants whose injections would not affect other buses due to the actions of voltage regulation devices. Three groups were identified based on the buss whose voltages are being regulated/controlled (buses 2 and 9): DG1 to DG3, DG4 to DG6, and DG7. Considering the wind profiles in each group (each profile with 150 generation levels), power factor settings (6 per DG plant), and demand (150 demand levels), $150 \times$ $(150 \times 6)^{3}=1.0935 \times 10^{11}$ scenarios were produced for DG1 to DG3 and DG4 to DG6, and $150 \times(150 \times 6)=$ 135,000 scenarios for DG7.

\section{A. Time Delays}

To coordinate the actions of multiple DG plants, different $T_{\text {delay }}$ and $T_{\text {hold }}$ values are allocated to each of them. As per section III-C, the seven DG plants are divided into three groups based on the corresponding feeders (i.e., DG1 to DG3 in group 1, DG4 to DG6 in group 2, and DG7 in group 3). Within the same group, the further the DG plant is from the OLTC, the higher the priority of curtailment. Therefore, considering a $T_{c}$ of two minutes and using (11) and (12), the corresponding $T_{\text {delay }}$ and $T_{\text {hold }}$ of DG1 to DG7 are $0,2,4,0,2$, 4, 0 and 6, 4, 2, 6, 4, 2, 2 minutes, respectively.

\section{B. Performance}

The performance of the proposed decentralized control algorithm for multiple DG plants and the centralized AC OPF is shown in Fig. 12 in terms of BS EN50160 compliance and capacity factor. Although the AC OPF has the ideal voltage compliance performance in which all seven DG plants achieve almost $100 \%$, the proposed decentralized approach reaches comparable results. As for the capacity factor, only DG2 resulted in a much lower performance than the AC OPF (from 0.34 to 0.09 p.u.). Nonetheless, the total energy harvested by the seven DG plants with the decentralized approach reached $2.0396 \mathrm{GWh}$; only $15.7 \%$ lower than that with the AC OPF (2.4194 GWh). This demonstrates that the proposed approach and time delays can be successfully used for multiple DG plants without the need of communication infrastructure.

\section{DISCUSSION}

This section discusses the implementation aspects of the proposed sensitivity approach and decentralized control algorithm in practice, and potential improvements.

\section{A. Scalability}

For the proposed sensitivity approach, the size of the distribution network (in terms of the number of nodes) does not per se affect the number of required demand/generation scenarios.
This is related to the number and diversity of DG plants and loads as well as to the granularity adopted for the scenarios. However, considerations based on the geography, technology, etc., scenarios can be the same for multiple locations (reducing the final number). For instance, within a given area, multiple wind farms can be considered to have the same generation behavior. Furthermore, considering the speed of current power flow algorithms and software packages, as well as available computing power (including parallel processing), dealing with a large number of scenarios should not represent a challenge. It is important to highlight that the computations related to the scenarios (power flows and surface fitting analysis) are carried out at a planning stage, i.e., before the control scheme is deployed. Therefore, it has no effect on the performance of the proposed decentralized control strategy.

Nonetheless, a given feeder with many DG plants with diverse behavior will result in $V, P$ and $Q$ surfaces with more voltage instances with a wider range. Although the Surface Fitting technique will still produce a fitting function that makes the most adequate compromises despite the more complex surface, the accuracy is likely to be affected. This is an unavoidable challenge of decentralized sensitivity approaches.

Even if the accuracy of the resulting fitting function is affected, the proposed decentralized voltage control approach still leads to promising results as demonstrated in section V-B. This is because, although the less accurate sensitivity coefficients will not necessarily achieve the desired outcome, the voltage is regularly checked so as to provide more opportunities to trigger further control actions. Therefore, the desired voltage target can be achieved asymptotically.

For the proposed control algorithm, the coordination of a large number of DG plants (to be locally controlled) would follow the same principles, i.e., using time delays for each of them and defining time delays according to their responses times and how fast problems need to be solved. However, if small-scale DG plants, such as domestic PV systems, are to be considered, then it is not practical to produce (and implement) different sensitivities and time delays for each of the hundreds or thousands of devices. In this case, the proposed methodology can be adapted to consider multiple small-scale DG plants in a given area as an aggregated DG plant. The resulting sensitivity could be then used by all the corresponding devices simultaneously. Moreover, the resulting time delays (for the aggregated DG plants, hence used by all the devices in the same area) would also provide adequate coordination of actions with upstream DG plants. This approach, however, requires further analysis and will be carried out as future work.

\section{B. Network Changes}

Once the fitting functions and time delays have been determined by the DNO (for specific network characteristics), they must be set by the DNO or DG owner at each DG site, i.e., locally for each DG plant participating in the scheme. Whilst this might be considered a time-consuming task, it can be comparable to the time required to deploy communication and control infrastructure in centralized approaches (that also involves each DG site). 
However, significant changes in load (e.g., a new substation), generation (e.g., a new DG plant), network parameters (e.g., asset replacement/upgrades) or topology (e.g., new configuration), do bring challenges. These are described below:

- Whilst new substations (new loads) do not change the time delays (as curtailment priorities will remain the same), the fitting functions will need to be updated;

- New generation, depending on its location, can affect both time delays and fitting functions;

- New impedances due to asset replacements or upgrades will affect only the fitting functions; and,

- Changes in topology will affect both time delays and fitting functions.

Significant changes in load, generation, and impedances may only occur after many months and even years and, therefore, the corresponding time delays and/or fitting functions at each DG plant can be updated manually. For topology changes, assuming these are planned and infrequent changes, then the updates can be done manually as well or based on a schedule. If changes in topology are unplanned and automatic (e.g., post-fault restoration), then a centralized or semi-centralized approach that uses communication would be needed; rendering inadequate any decentralized approach.

It is important to highlight that although manual updates of time delays and/or fitting functions might be considered a time-consuming task, it is achievable and not uncommon for other network elements. For instance, significant network changes could require adjusting the tap positions of off-load tap changers of distribution transformers (MV/LV). Depending on the area affected, there can be dozens to hundreds of them. But this is still done manually by DNOs.

\section{Other Implementation Aspects}

Depending on the DG technology, power factor control can require active power to be curtailed. However, technologies also exist that allow reactive power capabilities during full active power output [24]. Although this is likely to cost more, it might also bring more revenue to the DG owner. This is because the extra reactive power capabilities can significantly reduce voltage problems (that often happens during full active power output), reducing or avoiding the need of curtailment. Considering that revenues of most DG plants around the world come from $\mathrm{MWh}$, then minimizing curtailment means higher revenues. Nonetheless, it will be up to the DG owner to assess the corresponding cost-benefit from adopting the most suitable technology. This, however, might be a task to be done in conjunction with the DNO.

The proposed approach is still applicable even if there are DG plants with that do not participate in voltage control. The effects of those DG plants will still be captured when producing the demand/generation scenarios and, hence, the corresponding surfaces. However, if those DG plants that do not participate in voltage control have significantly used the "hosting capacity' of the network, then, even with the proposed approach successfully managing the voltages of the controllable DG plants, their resulting capacity factors might be significantly affected. At this stage, the decision to invest in these new DG plants is no longer technical (as problems could be solved) but economic. This is an issue that will also be faced by centralized approaches.

In the presence of locally-controlled voltage regulation elements such as capacitor banks, shunt reactors, OLTC-fitted transformers, etc., time delays should be tuned accordingly to ensure coordination along the feeder.

For unbalanced networks, the proposed sensitivity approach and control algorithm can still be applied but need to be adapted. Unbalanced power flows will allow the production of surfaces per phase and, thus, sensitivity functions per phase. The control logic should be adapted to calculate new settings for the DG plant based on its phase connection.

\section{CONCLUSIONS}

This paper presents a novel approach to produce voltage sensitivity coefficients using the surface fitting technique. Unlike methods available in the literature, the proposed approach is based solely on the knowledge of network characteristics, and only uses information at the bus of interest to update the sensitivity values, i.e., no remote monitoring is required.

Using the 16-bus UK GDS test network the proposed fitting function-based approach was compared to other two methods that require full network observability, the widelyused Jacobian matrix and perturb-and-observe. In terms of the coefficients, the values from the proposed approach, produced considering adequate demand/generation scenarios, were found to be close to those of the Jacobian matrix and better than the perturb-and-observe approach.

To assess the performance of the proposed approach in terms of its ability to manage voltages, a decentralized control logic for a single DG plant (that simultaneously caters for both the active and reactive power output) was adopted. Results highlight that the proposed sensitivity approach is more effective than the Jacobian matrix inverse and perturb-and-observe; also in terms of the capacity factor. Furthermore, a comparison with the benchmark AC OPF, demonstrates a high level of performance without the need of full network observability.

The robustness of the proposed sensitivity method was also investigated considering potential differences between the parameters of the adopted network model and the network to which DG settings applied in terms of different $R / X$ ratios and the upstream Thévenin equivalent reactance. The results confirm that the proposed approach continues to outperform the Jacobian matrix and have results similar to the AC OPF.

Finally, the more realistic and complex case of multiple DG plants was addressed adopting coordinated time delays. Comparisons with the AC OPF demonstrate that the proposed sensitivity approach and time delays can be successfully in such environment. Challenges related to scalability and implementation on large distribution networks with widespread DG plants were discussed, providing also a number of actions (e.g., scenario reduction, use of high performance computing, time delays/fitting function updates) that could be taken. Consequently, the combined use of the proposed voltage sensitivity approach and the decentralized controller represents an implementable, cost-effective solution to manage DG plants in 
distribution networks without the need of further communication infrastructure.

Ultimately, the usefulness of the proposed and other decentralized approaches, should be assessed on economic grounds (considering the resulting curtailment) as in some cases the extra benefits from centralized approaches might be justified.

\section{REFERENCES}

[1] R. A. Walling, et. al., "Summary of Distributed Resources Impact on Power Delivery Systems," IEEE Trans. Power Delivery, vol. 23, no. 3, pp. 1636-1644, Jul. 2008.

[2] L. F. Ochoa, C. J. Dent, G. P. Harrison, "Distribution Network Capacity Assessment: Variable DG and Active Networks", IEEE Trans. Pow. Syst, vol. 25, no. 1, pp. 87-95, Feb. 2010.

[3] S. W. Alnaser and L. F. Ochoa, "Adcanced network management systems: A risk-based AC OPF approach," IEEE Trans, Power, Syst., vol. 30, no. 1, pp. 409-418, Jan. 2015.

[4] T. Sansawatt, L. F. Ochoa, "Smart Decentralized Control of DG for Voltage and Thermal Constraint Management", IEEE Trans. Power Syst., vol. 27, no. 3, pp. 1637-1645, Aug. 2012.

[5] O. Frédéric, P. Aristidou, D. Ernst and T. V. Cutsem, "Active management of low-voltage networks for mitigating over voltages due to photovoltaic units," IEEE Transactions on Smart Grid, vol. 7, no.2, pp. 926936, 2016.

[6] J. W. Smith, W. Sunderman and R. Dugan, "Smart inverter volt/var control functions for high penetration of PV on distribution systems," Power Systems Conference and Exposition (PSCE), 2011 IEEE/PES. IEEE, 2011.

[7] M. C. F. Donkers, W. P. M. H. Heemels, D. Bernardini, A. Bemporad, and V. Shneer. "Stability analysis of stochastic networked control systems," Automatica, vol. 48, no. 5, pp. 917-925, 2012.

[8] D. Yue, Q. Han, and J. Lam, "Network-based robust $H_{\infty}$ control of systems with uncertainty," Automatica, vol. 41, no. 6, pp. 999-1007, 2005.

[9] A. Keane, L. F. Ochoa, C. L. T. Borges, G. W. Ault, A. D. AlarconRodriguez, R. A. F. Currie, F. Pilo, C. Dent, and G. P. Harrison, "Stateof-the-Art Techniques and Challenges Ahead for Distributed Generation Planning and Optimization," IEEE Trans. Power Syst., vol. 28, pp. 1493-1502, 2013.

[10] P. N. Vovos, A. E. Kiprakis, A. R. Wallace, and G. P. Harrison, "Centralized and distributed voltage control: Impact on distributed generation penetration," IEEE Trans. Power. Syst., vol. 22, no. 1, pp. 476-483, Feb. 2007.

[11] K. E. A. Plytaria, I. N. K. Lysikatos, P. S. Georgilakis, N. D. Hatziargyriou, "Distributed and decentralized voltage control of smart distribution network: models, method, and future research," IEEE Trans. On Smart Grid, issue 99, Mar. 2017.

[12] M. E. Elkhatib, R. E. Shatshat, and M. M. A. Salama, "Decentralized reactive power control for advanced distribution automation systems," IEEE Trans. Smart Grid, vol. 3, no. 3, pp. 1482-1490, Sep. 2012.

[13] M. Wolter, S. Brenner, T. Isermann, and L. Hofmann, "Application of adaptive agents in decentralized energy management systems for the purpose of voltage stability in distribution grids," in North American Power Symposium (NAPS), pp. 1-5, 2009.

[14] B. Seal, "Common functions for smart inverters: 4th Edition", EPRI, California, Tech. Rep. 3002008217, Dec. 2016.

[15] A. O'Connell, and A. Keane. "Volt-var curves for photovoltaic inverters in distribution systems," IET Generation, Transmission \& Distribution, pp. 730-739, 2016

[16] G. Valverde and T. V. Custsem, "Model Predictive Control of Voltages in Active Distribution Networks", Smart Grid, IEEE Trans. Smart Grid, pp. 2152-2161, Feb. 2013

[17] Q. Zhou and J. Bialek, "Simplified calculation of voltage and loss sensitivity factors in distribution networks", in Proc. 16th Power Syst. Compute. Conf. (PSCC), Glasgow, U.K., 2008.

[18] C. Murphy, A. Keane, "Optimised voltage control for distributed generation," PowerTech, 2015 IEEE Eindhoven, Jul. 2015.

[19] S. Weckx, R. D’Hulst, J. Driesen, "Voltage Sensitivity Analysis of a Laboratory Distribution Grid With Incomplete Data," IEEE Trans. Smart Grid, vol. 6, no. 3, pp. 1271-1280, Apr. 2015.

[20] P. Dierckx, Curve and Surface Fitting with Splines. Monographs on Numerical Analysis. Clarendon Press, Oxford, 1993.
[21] J. Miles, M. Shevlin, "Applying Regression and Correlation: A Guide for Students and Researchers", SAGE Publications, Mar. 2001.

[22] L. Kane, G. Ault, "A review and analysis of renewable energy curtailment schemes and Principles of Access: Transitioning towards business as usual," Energy Policy, Vol. 72, Sep. 2014, pp. 67-77.

[23] Distributed Generation and Sustainable Electrical Energy Centre, United Kingdom Generic Distribution System (UKGDS). [Online] Available: http://github.com/sedg/ukgds.

[24] A. Ellis, R. Nelson, E. Von Engeln, "Reactive power performance requirements for wind and solar plants", 2012 IEEE/PES General Meeting, pp.1-8, 2012.

[25] Curve Fitting Toolbox User's Guide. [Online] Available: https://www.fkit.unizg.hr/_download/repository/MS_CFT.pdf

[26] R. Dugan and T. E. McDermott, "An open source platform for collaborating on smart grid research," in Proc. IEEE/PES General Meeting 2011, pp. 1-7, 2011.

[27] Visual Basic [Online] Available: https://msdn.microsoft.com/library/2x7h1hfk.aspx

[28] J. Bisschop and M. Roelofs, "AIMMS - The user's guide," Paragon Decision Technology, 2006.

[29] UK Department of Trade and Industry, The electricity supply, quality and continuity regulations 2002, Sep. 2002. 